\title{
Development and validation of a prognostic nomogram for predicting in-hospital mortality of COVID-19: a multicenter retrospective cohort study of 4086 cases in China
}

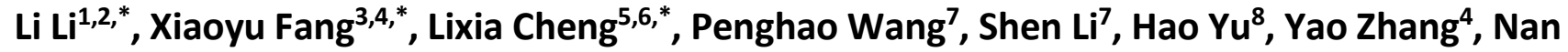

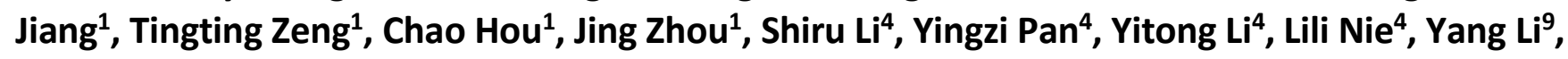 \\ Qidi Sun ${ }^{2,5}$, Hong Jia ${ }^{3}$, Mengxia $\mathrm{Li}^{6,10}$, Guoqiang Cao ${ }^{1,2}$, Xiangyu Ma ${ }^{4}$ \\ ${ }^{1}$ Department of Respiratory Medicine, Daping Hospital, Army Medical University, Former Third Military Medical \\ University, Chongqing, China \\ ${ }^{2}$ Wuhan Huoshenshan Hospital, Wuhan, China \\ ${ }^{3}$ College of Public Health, Southwest Medical University, Luzhou, Sichuan, China \\ ${ }^{4}$ Department of Epidemiology, College of Preventive Medicine, Army Medical University, Former Third Military \\ Medical University, Chongqing, China \\ ${ }^{5}$ Daping Hospital, Army Medical University, Former Third Military Medical University, Chongqing, China \\ ${ }^{6}$ Wuhan Taikang Tongji Hospital, Wuhan, China \\ ${ }^{7}$ The Second Clinical College, Chongqing Medical University, Chongqing, China \\ ${ }^{8}$ Department of Endocrinology, General Hospital of Northern Theater Command, Shenyang, Liaoning, China \\ ${ }^{9} \mathrm{NCO}$ School of Army Medical University (Former Third Military Medical University), Shijiazhuang, Hebei, China \\ ${ }^{10}$ Department of Oncology, Daping Hospital, Army Medical University, Former Third Military Medical University, \\ Chongqing, China \\ *Equal contribution
}

Correspondence to: Xiangyu Ma, Guoqiang Cao; email: xymacq@hotmail.com, https://orcid.org/0000-0001-7967-3950; cgq1963@163.com, https://orcid.org/0000-0001-8648-7654

Keywords: COVID-19, SARS-Cov-2, coronavirus, nomogram, survival

Received: October 23, $2020 \quad$ Accepted: December 23, $2020 \quad$ Published: February 9, 2021

Copyright: (C) $2021 \mathrm{Li}$ et al. This is an open access article distributed under the terms of the Creative Commons Attribution License (CC BY 3.0), which permits unrestricted use, distribution, and reproduction in any medium, provided the original author and source are credited.

\section{ABSTRACT}

To establish an effective nomogram for predicting in-hospital mortality of COVID-19, a retrospective cohort study was conducted in two hospitals in Wuhan, China, with a total of 4,086 hospitalized COVID-19 cases. All patients have reached therapeutic endpoint (death or discharge). First, a total of 3,022 COVID-19 cases in Wuhan Huoshenshan hospital were divided chronologically into two sets, one (1,780 cases, including 47 died) for nomogram modeling and the other (1,242 cases, including 22 died) for internal validation. We then enrolled 1,064 COVID-19 cases (29 died) in Wuhan Taikang-Tongji hospital for external validation. Independent factors included age (HR for per year increment: 1.05), severity at admission (HR for per rank increment: 2.91), dyspnea (HR: 2.18), cardiovascular disease (HR: 3.25), and levels of lactate dehydrogenase (HR: 4.53), total bilirubin (HR: 2.56), blood glucose (HR: 2.56), and urea (HR: 2.14), which were finally selected into the nomogram. The Cindex for the internal resampling $(0.97,95 \% \mathrm{Cl}: 0.95-0.98)$, the internal validation $(0.96,95 \% \mathrm{Cl}: 0.94-0.98)$, and the external validation $(0.92,95 \% \mathrm{Cl}: 0.86-0.98)$ demonstrated the fair discrimination ability. The calibration plots showed optimal agreement between nomogram prediction and actual observation. We established and validated a novel prognostic nomogram that could predict in-hospital mortality of COVID-19 patients. 


\section{INTRODUCTION}

Since being publicly characterized as a pandemic by the World Health Organization on March $11^{\text {th }}, 2020$, the coronavirus disease 2019 (COVID-19) has become an urgent threat to global public health [1]. The outbreak of COVID-19 led to a significant increase in demand for hospital beds and medical equipment, and several countries have been confronted with a critical care crisis [2]. Therefore, it is urgently needed to set up clinical prediction models for COVID-19 mortality to stratify the most vulnerable patients, to provide them with the best possible care while mitigating the burden on the whole healthcare system.

A number of studies have identified risk factors associated with poor outcomes in COVID-19 univariate/ multivariate analyses [3]. For example, older age, comorbidities, higher sequential organ failure assessment (SOFA) score, lower lymphocyte count and increased d-dimer have been reported to be associated with an increased risk of death for COVID-19 patients [4-6]. Besides, several models have been developed to assist in the prognosis of COVID-19 mortality, including nomogram [7], decision tree [8], score system [9], online tools [10], and computed tomography based scoring rule [11], most of which are still in preprint. However, as pointed out by a recent systematic review [12], despite 23 prognostic models to predict mortality risk in patients with COVID-19 having been reported, none was recommended for use in practice due to several limitations. First, some studies suffered from severe sampling bias which was caused by excluding participants who didn't reach an endpoint (recovered or died). Second, limited sample size, varied length of follow-up, highly subjective predictors, and lack of external validation. Third, the calibration of the models was rarely assessed. Fourth, the guidelines of transparent reporting of a multivariable prediction model for individual prognosis or diagnosis (TRIPOD) were not complied with, and prediction model risk of bias assessment tool (PROBAST) showed these studies were at high risk of bias.

In the current study, we aimed to establish an effective prognostic nomogram for predicting in-hospital mortality of COVID-19 patients. We presented the details of all 4,086 patients with laboratory-confirmed COVID-19 admitted to the two designated hospitals in Wuhan, Huoshenshan Hospital and Taikang Tongji Hospital, as of April $10^{\text {th }}$. The prognostic nomogram was validated by internal 1,000 bootstrap resampling, internal and external validation cohorts. The performance of the nomogram was measured by Harrel concordance index (C-index) for discrimination and the calibration plot for calibration.

\section{RESULTS}

\section{Characteristics of the COVID-19 patients}

Table 1 presents the demographic and clinical characteristics of the included COVID-19 patients in the development cohort and the validation cohort. Of the 4,086 COVID-19 cases, 98 (2.4\%) died. Of the 3,988 discharged patients, the median duration of hospitalization was 14 days (IQR: 9-20). For the 98 died, the median duration from admission to death was 9 days (IQR: 5-17). Males accounted for $50.0 \%(n=2,043)$ of the total cases, and the median age was 61 (IQR: 50 to 69). For the severity at admission, $48(1.2 \%)$ were classified as mild, 2,882 (70.5\%) as ordinary, $1076(26.3 \%)$ as severe, and $80(2.0 \%)$ as critical. The top five symptoms were fever $(70.6 \%)$, cough $(69.2 \%)$, fatigue $(5=54.7 \%)$, anorexia (52.5\%), and short breath (41.3\%), respectively. The top five coexisting disorders were hypertension $(31.7 \%)$, diabetes $(14.5 \%)$, cardiovascular disease $(10.5 \%)$, coronary heart disease $(7.3 \%)$, and Chronic liver disease (5.3\%), respectively.

\section{Prognostic factors of in-hospital mortality of COVID-19}

Table 2 presents the results of the univariate and multivariate $\mathrm{COX}$ proportional hazards regression analysis of in-hospital mortality of COVID-19 in the development cohort of 1780 cases (47 deaths). First, univariate Cox regression analysis was used to explore the potential prognostic predictors (including demographic, symptoms at admission, coexisting disorders, and Laboratory findings at admission), and revealed 29 of the 48 predictors was significantly associated with in-hospital mortality of COVID-19. The HRs ranged from 1.10 (1.07-1.13) for per year increment of age, to 23.11 (9.05$58.98)$ for lactate dehydrogenase $(\geq 250 \mathrm{U} / \mathrm{L})$. The top five prognostic predictors were lactate dehydrogenase $(\geq 250$ U/L, HR:23.11), C-reactive protein (>10mg/l, HR: 12.34), D-dimer ( $\geq 0.5 \mathrm{mg} / \mathrm{L}, \mathrm{HR}: 11.29)$, urea $(>7.5 \mathrm{mmol} / \mathrm{L}$, HR: 9.93), and cardiovascular disease (HR: 8.87), respectively.

\section{Construction of the prognostic nomogram}

According to the AIC selection procedure, 8 independent prognostic predictors (Figure 1), including age (HR for per year increment: 1.05; 95\% CIs: 1.01-1.09; $\mathrm{P}=0.003$ ), severity at admission (HR for per rank increment: $2.91 ; 95 \%$ CIs: 1.71-4.97; $\mathrm{P}<0.001)$, dyspnea (HR: 2.18; 95\% CIs: 1.11-4.27; $\mathrm{P}=0.023$ ), cardiovascular disease (HR: 3.25; 95\% CIs: 1.71-6.17; $\mathrm{P}<0.001$ ), lactate dehydrogenase (HR: 4.53 ; $95 \%$ CIs: 1.62-1.63; $\mathrm{P}=0.004$ ), total bilirubin (HR: $2.56 ; 95 \%$ CIs: 1.34-4.90; $\mathrm{P}=0.014$ ), blood glucose (HR: $2.56 ; 95 \%$ 
Table 1. Demographic and clinical characteristics of the included COVID-19 patients.

\begin{tabular}{|c|c|c|c|c|}
\hline Variables & Total $(\mathrm{N}=\mathbf{4 0 8 6})$ & $\begin{array}{c}\text { Development } \\
\text { cohort }(\mathbf{N}=\mathbf{1 7 8 0})\end{array}$ & $\begin{array}{c}\text { Internal validation } \\
\quad(\mathrm{N}=1242)\end{array}$ & $\begin{array}{c}\text { External } \\
\text { validation } \\
(\mathrm{N}=1064) \\
\end{array}$ \\
\hline Death & $98(2.4 \%)$ & $47(2.6 \%)$ & $22(1.8 \%)$ & $29(2.7 \%)$ \\
\hline Gender, male & $2043(50.0 \%)$ & $914(51.3 \%)$ & $627(50.5 \%)$ & $502(47.2 \%)$ \\
\hline Age (Years old), median (IQR) & $61(50-69)$ & $60(49-68)$ & $60(49-69)$ & $62(51-71)$ \\
\hline $0-14$ & $3(0.1 \%)$ & $2(0.1 \%)$ & - & $1(0.1 \%)$ \\
\hline $15-49$ & $1003(24.5 \%)$ & $444(24.9 \%)$ & $317(25.5 \%)$ & $242(22.7 \%)$ \\
\hline $50-64$ & $1505(36.8 \%)$ & $690(38.8 \%)$ & $465(37.4 \%)$ & $350(32.9 \%)$ \\
\hline$\geq 65$ & $1575(38.5 \%)$ & $644(36.2 \%)$ & $460(37.0 \%)$ & $471(44.3 \%)$ \\
\hline \multicolumn{5}{|l|}{ Severity at admission } \\
\hline Mild & $48(1.2 \%)$ & $16(0.9 \%)$ & $15(1.2 \%)$ & $17(1.6 \%)$ \\
\hline Ordinary & $2882(70.5 \%)$ & $1260(70.8 \%)$ & $903(72.7 \%)$ & $719(67.6 \%)$ \\
\hline Severe & $1076(26.3 \%)$ & $478(26.9 \%)$ & $312(25.1 \%)$ & $286(26.9 \%)$ \\
\hline Critical & $80(2.0 \%)$ & $26(1.5 \%)$ & $12(1.0 \%)$ & $42(3.9 \%)$ \\
\hline Respiratory rate (times) & $20(18-21)$ & $20(19-21)$ & $20(19-21)$ & $16(11-23)$ \\
\hline \multicolumn{5}{|l|}{ Symptoms - no. (\%) } \\
\hline Fever & $2885(70.6 \%)$ & $1389(78.0 \%)$ & $833(67.2 \%)$ & $663(62.3 \%)$ \\
\hline Cough & $2827(69.2 \%)$ & $1346(76.2 \%)$ & $841(67.9 \%)$ & $640(60.2 \%)$ \\
\hline Fatigue & $2237(54.7 \%)$ & $1128(63.9 \%)$ & $633(51.1 \%)$ & $476(44.7 \%)$ \\
\hline Anorexia & $2145(52.5 \%)$ & $1047(59.3 \%)$ & $644(51.9 \%)$ & $454(42.7 \%)$ \\
\hline Short breath & $1686(41.3 \%)$ & $931(52.7 \%)$ & $527(42.5 \%)$ & $220(20.7 \%)$ \\
\hline Myalgia & $1093(36.7 \%)$ & $675(38.3 \%)$ & $329(26.6 \%)$ & $89(8.4 \%)$ \\
\hline Chest tight & $1179(28.9 \%)$ & $589(33.3 \%)$ & $292(23.6 \%)$ & $298(28.0 \%)$ \\
\hline Expectoration & $728(17.8 \%)$ & $313(17.7 \%)$ & $214(17.2 \%)$ & $201(18.9 \%)$ \\
\hline Dyspnea & $327(8.0 \%)$ & $112(6.3 \%)$ & $81(6.5 \%)$ & $134(12.6 \%)$ \\
\hline Diarrhea & $268(6.6 \%)$ & $121(6.8 \%)$ & $65(5.2 \%)$ & $82(7.7 \%)$ \\
\hline Sore throat & $227(5.6 \%)$ & $80(4.5 \%)$ & $48(3.9 \%)$ & $99(9.3 \%)$ \\
\hline Nausea & $121(3.0 \%)$ & $58(3.3 \%)$ & $30(2.4 \%)$ & $33(3.1 \%)$ \\
\hline Dizziness & $107(2.6 \%)$ & $47(2.7 \%)$ & $29(2.3 \%)$ & $31(2.9 \%)$ \\
\hline Headache & $104(2.5 \%)$ & $53(3.0 \%)$ & $17(1.4 \%)$ & $34(3.2 \%)$ \\
\hline Vomiting & $104(2.5 \%)$ & $51(2.9 \%)$ & $21(1.7 \%)$ & $32(3.0 \%)$ \\
\hline Chill & $94(2.3 \%)$ & $38(2.2 \%)$ & $21(1.7 \%)$ & $35(3.3 \%)$ \\
\hline Hemoptysis & $26(0.6 \%)$ & $11(0.6 \%)$ & $10(0.8 \%)$ & $5(0.5 \%)$ \\
\hline \multicolumn{5}{|l|}{ Coexisting disorders - no. $(\%)$} \\
\hline Hypertension & $1294(31.7 \%)$ & $535(30.1 \%)$ & $385(31.0 \%)$ & $374(35.2 \%)$ \\
\hline Diabetes & $592(14.5 \%)$ & $260(14.6 \%)$ & $168(13.5 \%)$ & $164(15.4 \%)$ \\
\hline Cardiovascular disease & $427(10.5 \%)$ & $152(8.5 \%)$ & $129(10.4 \%)$ & $146(13.7 \%)$ \\
\hline Coronary heart disease & $300(7.3 \%)$ & $114(6.4 \%)$ & $96(7.7 \%)$ & $90(8.5 \%)$ \\
\hline Chronic liver disease & $215(5.3 \%)$ & $109(6.1 \%)$ & $84(6.8 \%)$ & $22(2.1 \%)$ \\
\hline Cerebrovascular disease & $195(4.8 \%)$ & $53(3.0 \%)$ & $63(5.1 \%)$ & $79(7.4 \%)$ \\
\hline Respiratory disease & $175(4.3 \%)$ & $36(2.0 \%)$ & $53(4.3 \%)$ & $86(8.1 \%)$ \\
\hline Chronic kidney disease & $123(3.0 \%)$ & $43(2.4 \%)$ & $52(4.2 \%)$ & $28(2.6 \%)$ \\
\hline Tumor & $92(2.3 \%)$ & $35(2.0 \%)$ & $42(3.4 \%)$ & $15(1.4 \%)$ \\
\hline Bronchitis & $81(2.0 \%)$ & $19(1.1 \%)$ & $33(2.7 \%)$ & $29(2.7 \%)$ \\
\hline COPD & $60(1.5 \%)$ & $15(0.8 \%)$ & $15(1.2 \%)$ & $30(2.8 \%)$ \\
\hline \multicolumn{5}{|l|}{ Laboratory findings } \\
\hline White-cell count $\left(\times 10^{9} / \mathrm{L}\right)$ & $5.8(4.7-7.1)$ & $5.7(4.6-7.1)$ & $5.7(4.7-7.0)$ & $5.8(4.8-7.0)$ \\
\hline
\end{tabular}




\begin{tabular}{lcccc} 
Neutrophil count $\left(\times 10^{9} / \mathrm{L}\right)$ & $3.52(2.68-4.73)$ & $3.52(2.67-4.80)$ & $3.46(2.72-4.48)$ & $3.51(2.66-4.89)$ \\
Lymphocyte count $\left(\times 10^{9} / \mathrm{L}\right)$ & $1.52(1.11-1.92)$ & $1.43(1.04-1.83)$ & $1.56(1.17-1.92)$ & $1.65(1.23-2.11)$ \\
Monocyte count $\left(\times 10^{9} / \mathrm{L}\right)$ & $0.45(0.35-0.58)$ & $0.43(0.34-0.55)$ & $0.43(0.34-0.54)$ & $0.53(0.41-0.69)$ \\
Platelet count $\left(\times 10^{9} / \mathrm{L}\right)$ & $223(180-274)$ & $232(183-290)$ & $213(178-261)$ & $219(177-265)$ \\
C-reactive protein $(\mathrm{mg} / \mathrm{l})$ & $2.07(0.60-8.66)$ & $2.80(0.97-12.10)$ & $1.89(0.66-6.15)$ & $0.50(0.50-6.33)$ \\
D-dimer, mg/L & $0.41(0.19-0.86)$ & $0.42(0.19-0.86)$ & $0.42(0.23-0.93)$ & $0.24(0.10-0.68)$ \\
Alanine aminotransferase, U/L & $22.5(14.3-37.2)$ & $24.3(15.3-40.9)$ & $20.8(13.6-33.8)$ & $21.6(13.8-35.2)$ \\
Aspartate aminotransferase, U/L & $20.0(15.9-26.9)$ & $20.2(15.9-27.7)$ & $19.1(15.4-25.2)$ & $21.3(17.0-27.8)$ \\
Albumin, g/L & $37.8(34.7-40.4)$ & $36.8(33.8-39.5)$ & $38.9(36.2-41.2)$ & $38.1(34.5-40.8)$ \\
Total bilirubin, $\mu$ mol/L & $9.9(7.6-12.6)$ & $9.3(7.2-12.1)$ & $9.8(7.4-12.8)$ & $10.6(8.6-13.2)$ \\
Blood glucose, mmol/L & $5.02(4.55-5.77)$ & $4.86(4.44-5.65)$ & $4.90(4.50-5.63)$ & $5.36(4.91-6.05)$ \\
Urea, mmol/L & $4.58(3.73-5.55)$ & $4.28(3.50-5.33)$ & $4.48(3.69-5.71)$ & $4.99(4.28-5.75)$ \\
Creatinine, $\mu$ mol/L & $62.2(52.7-74.4)$ & $64.0(54.7-75.2)$ & $64.4(55.6-76.0)$ & $54.7(45.3-68.0)$ \\
Lactate dehydrogenase, U/L & $171.5(144.7-208.0)$ & $182.7(155.6-229.0)$ & $175.4(141.2-221.2)$ & $171.5(149.7-202.1)$ \\
\hline
\end{tabular}

All continuous variables were presented using the median/interquartile range (IQR) values.

Table 2. Univariate and multivariate COX proportional hazards regression analysis of fetal outcome of COVID-19 in the development cohort.

\begin{tabular}{|c|c|c|c|c|}
\hline \multirow{2}{*}{ Variables } & \multicolumn{2}{|c|}{ Univariate COX analysis } & \multicolumn{2}{|c|}{ Multivariate COX analysis } \\
\hline & HR (95\% CIs) & $P$ value & HR (95\% CIs) & $P$ value \\
\hline Gender, male & $2.00(1.08-3.69)$ & 0.027 & - & - \\
\hline Age, per year increment & $1.10(1.07-1.13)$ & $<0.001$ & $1.05(1.01-1.09)$ & 0.003 \\
\hline Severity at admission, per rank & $8.50(5.37-13.53)$ & $<0.001$ & $2.91(1.71-4.97)$ & $<0.001$ \\
\hline Respiratory rate (times), $\geq 30$ & $7.59(3.29-17.50)$ & $<0.001$ & - & - \\
\hline \multicolumn{5}{|l|}{ Symptoms } \\
\hline Fever & $4.25(1.03-17.56)$ & 0.045 & - & - \\
\hline Cough & $1.54(0.60-3.96)$ & 0.371 & - & - \\
\hline Fatigue & $1.24(0.60-2.57)$ & 0.561 & - & - \\
\hline Anorexia & $1.71(0.78-3.78)$ & 0.183 & - & - \\
\hline Short breath & $1.84(0.97-3.49)$ & 0.064 & - & - \\
\hline Myalgia & $1.17(0.61-2.25)$ & 0.628 & - & - \\
\hline Chest tight & $1.37(0.71-2.62)$ & 0.346 & - & - \\
\hline Expectoration & $0.83(0.36-1.89)$ & 0.651 & - & - \\
\hline Diarrhea & $0.61(0.15-2.54)$ & 0.495 & - & - \\
\hline Dyspnea & $5.59(3.01-10.39)$ & $<0.001$ & $2.18(1.11-4.27)$ & 0.023 \\
\hline Sore throat & $1.39(0.33-5.78)$ & 0.652 & - & - \\
\hline Nausea & $2.29(0.70-7.46)$ & 0.171 & - & - \\
\hline Vomiting & $1.74(0.42-7.23)$ & 0.448 & - & - \\
\hline Chest pain & $0.87(0.12-6.38)$ & 0.891 & - & - \\
\hline Dizziness & $1.80(0.44-7.45)$ & 0.417 & - & - \\
\hline \multicolumn{5}{|l|}{ Coexisting disorders } \\
\hline Hypertension & $3.14(1.74-5.68)$ & $<0.001$ & - & - \\
\hline Diabetes & $1.80(0.95-3.42)$ & 0.074 & - & - \\
\hline Coronary heart disease & $4.92(2.55-9.49)$ & $<0.001$ & - & - \\
\hline Cardiovascular disease & $8.87(4.99-15.77)$ & $<0.001$ & $3.25(1.71-6.17)$ & $<0.001$ \\
\hline Cerebrovascular disease & $4.44(1.97-10.00)$ & $<0.001$ & - & - \\
\hline
\end{tabular}


Tumor

Bronchitis

COPD

Respiratory disease

Chronic kidney disease

Chronic liver disease

\section{Laboratory findings}

White-cell count $\left(\times 10^{9} / \mathrm{L}\right)$

$$
\begin{aligned}
& <4 \\
& 4-10
\end{aligned}
$$$$
\geq 10
$$

Neutrophil count $\left(\times 10^{9} / \mathrm{L}\right)$

$$
\begin{aligned}
& <1.8 \\
& 2.8-3.6 \\
& \geq 3.6
\end{aligned}
$$

Lymphocyte count, $<1.5 \times 10^{9} / \mathrm{L}$

Monocyte count, $\geq 0.6 \times 10^{9} / \mathrm{L}$

Platelet count $<150 \times 10^{9} / \mathrm{L}$

C-reactive protein, $>10 \mathrm{mg} / 1$

D-dimer, $\geq 0.5 \mathrm{mg} / \mathrm{L}$

Alanine aminotransferase, $>40 \mathrm{U} / \mathrm{L}$

Aspartate aminotransferase, $>40 \mathrm{U} / \mathrm{L}$

Albumin, <40 g/L

Total bilirubin, $>17.1 \mu \mathrm{mol} / \mathrm{L}$

Blood glucose, $>6.1 \mathrm{mmol} / \mathrm{L}$

Urea, $>7.5 \mathrm{mmol} / \mathrm{L}$

Creatinine, $\geq 133 \mu \mathrm{mol} / \mathrm{L}$

Alkaline phosphatase, $>135 \mathrm{U} / \mathrm{L}$

$\gamma$-glutamyl transpeptidase, $>45 \mathrm{U} / \mathrm{L}$

Creatine kinase, $\geq 200 \mathrm{U} / \mathrm{L}$

Lactate dehydrogenase, $\geq 250 \mathrm{U} / \mathrm{L}$
$0.60(0.08-4.42) \quad 0.618$

$1.00(0.14-7.33) \quad 0.996$

$5.17(1.25-21.39) \quad 0.023$

$2.20(0.68-7.19) \quad 0.190$

$5.06(2.14-11.97) \quad<0.001$

$2.38(1.11-5.14) \quad 0.027$

\section{Referent}

$0.78(0.30-2.04) \quad 0.611$

$4.42(1.58-12.33) \quad 0.005$

Referent

$0.30(0.06-1.54) \quad 0.149$

$1.60(0.38-6.70) \quad 0.518$

$5.84(2.08-16.43) \quad 0.001$

$1.28(0.65-2.54) \quad 0.473$

$4.46(2.49-8.02) \quad<0.001$

$12.34(4.84-31.51) \quad<0.001$

$11.29(3.48-36.63) \quad<0.001$

$1.46(0.79-2.69) \quad 0.225$

$3.18(1.70-5.97) \quad<0.001$

$7.34(1.00-53.66) \quad 0.049$

$4.50(2.40-8.43) \quad<0.001$

$2.25(1.18-4.30) \quad 0.014$

$5.98(3.25-10.99) \quad<0.001$

$2.56(1.34-4.90) \quad 0.004$

$9.93(5.50-17.95) \quad<0.001$

$2.14(1.15-3.97) \quad 0.016$

$4.50(1.38-14.67) \quad 0.012$

$4.15(1.88-9.17) \quad<0.001$

$2.46(1.08-5.60) \quad 0.032$

$5.92(2.64-13.29) \quad<0.001$

$23.11(9.05-58.98) \quad<0.001$

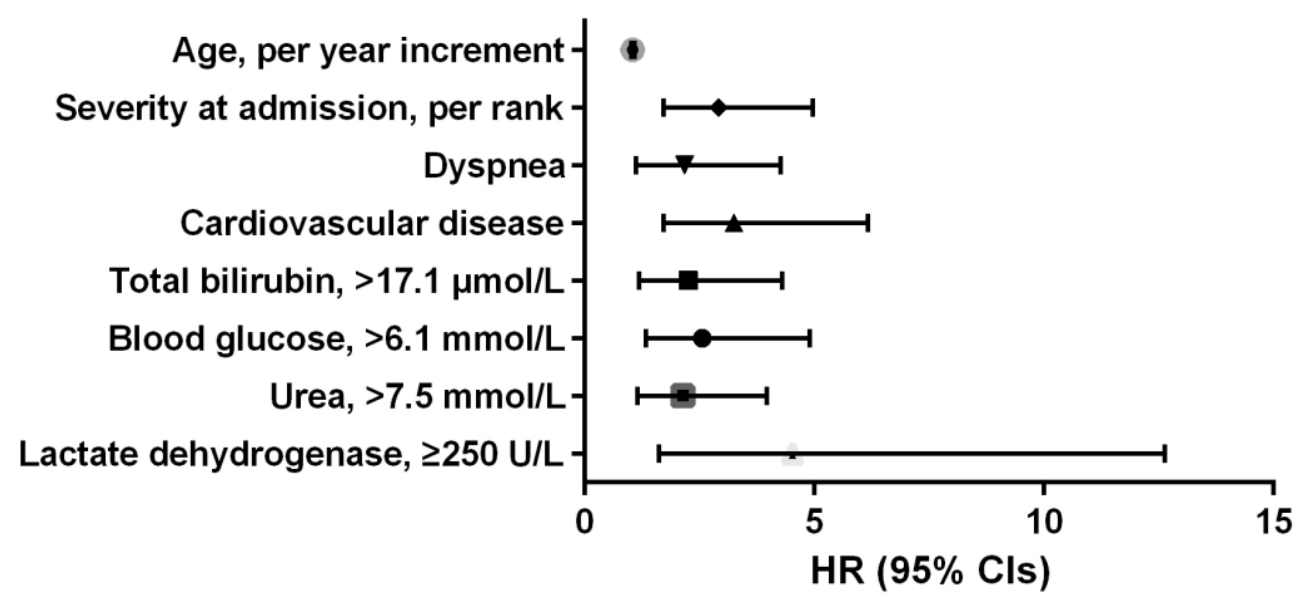

Figure 1. Independent prognostic predictors associated with in-hospital mortality of COVID-19 in the development cohort. 
CIs: $1.34-4.90 ; \mathrm{P}=0.004)$, and urea (HR: $2.14 ; 95 \%$ CIs: 1.15-3.97; $\mathrm{P}=0.016)$ were selected for the construction of the prognostic nomogram (Figure 2). The C-index for inhospital mortality prediction of COVID-19 was 0.97 (95\% CI, 0.95 to $0.98, \mathrm{P}<0.001$ ). The calibration plot for the probability of survival at 5-, 15-, and 30-days after admission showed an optimal agreement between the prediction by nomogram and actual observation (Figure 3 ).

\section{Validation and calibration of the prognostic nomogram}

An internal validation of the prognostic nomogram was conducted in 1,242 COVID-19 cases (22 deaths), while an external validation was conducted in 1,064 COVID19 cases from Taikang-Tongji hospital (29 deaths). The C-index for in-hospital mortality prediction of COVID19 was 0.96 (95\% CI, 0.94 to $0.98, \mathrm{P}<0.001)$ for the internal validation. For the external validation, the Cindex for in-hospital mortality prediction of COVID-19 was $0.92\left(95 \% \mathrm{CI}, 0.86\right.$ to $\left.0.98, \mathrm{P}=9.7 \times 10^{-38}\right)$. The calibration plot of both internal validation (Figure 4) and external validation (Figure 5) for the probability of survival at 5-,15-, and 30-days after admission also showed an optimal agreement between the prediction by nomogram and actual observation.

\section{DISCUSSION}

In the current study, we established a prognostic nomogram to predict in-hospital mortality of COVID-19 based on eight independent factors including age, severity at admission, cardiovascular disease, and levels of lactate dehydrogenase, total bilirubin, blood glucose, and urea in a large and well-described population of 4, 086 patients. The prognostic nomogram has been validated by internal 1,000 bootstrap resampling, an internal validation, as well as an external validation cohort, maintaining an adequate calibration and discrimination capacity. To our knowledge, this is the largest and most comprehensive study which aims to establish and validate an effective prognostic nomogram for predicting in-hospital mortality of COVID-19 patients to date.

Vague reporting of study population, as well as substantial sampling bias and limited sample size are main obstacles preventing clinical use of previous prognostic models for COVID-19 [12]. The wide variation is mainly caused by excluding participants who did not reach endpoints (neither recovered nor died) and difficulty of data collection under epidemic conditions, with death percentage varying between $1 \%$ and $59 \%$ in those studies that developed prognostic models to predict mortality [12]. This will inevitably yield a highly selected and biased sample and restrain application of those models. In the current study, we included all patients that had been treated from 2 designated hospitals in Wuhan, with a relatively large cohort of 4, 086 in-hospital patients with $100 \%$ ascertainment of endpoints (recovered or died). The clinical characteristics of these patients were welldescribed, and they serve as a good representation of general in-hospitalized COVID-19 patients.

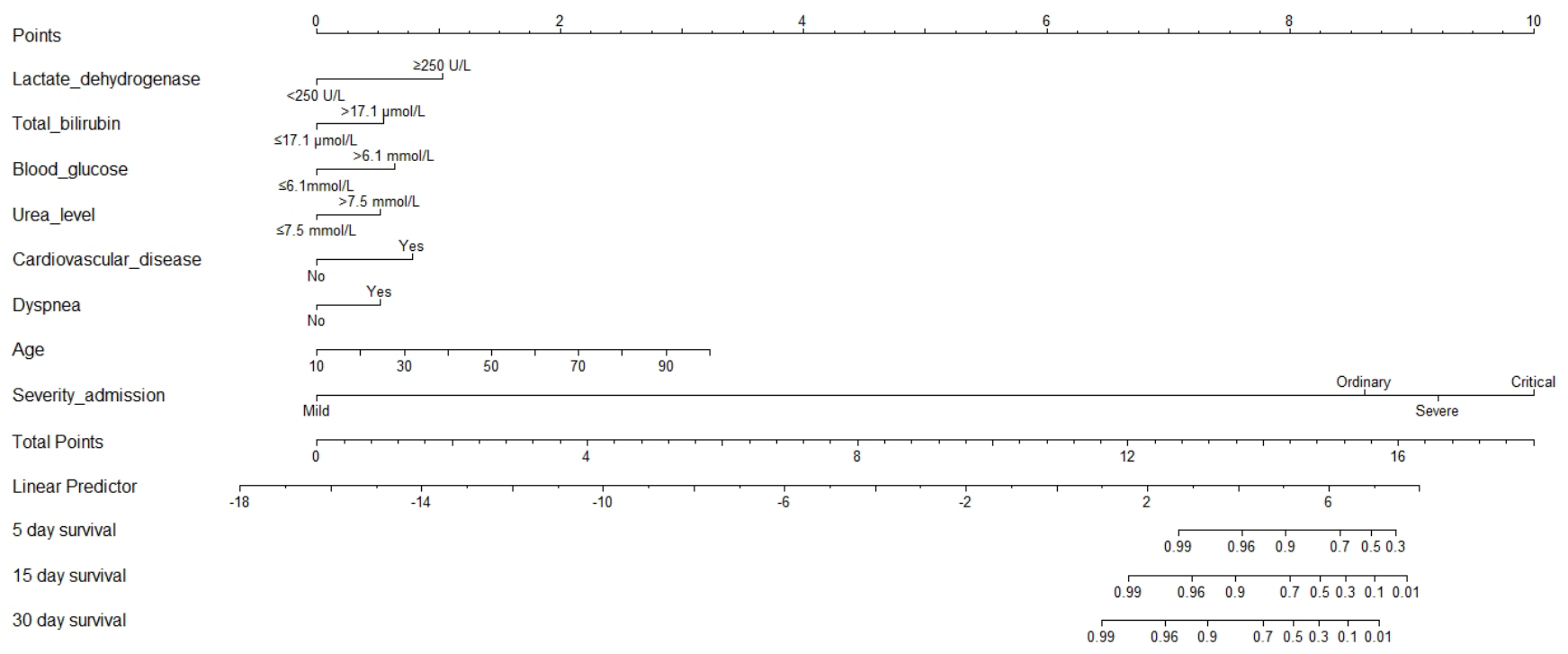

Figure 2. Prognostic nomogram for COVID-19. The nomogram variables include age, disease severity at admission, dyspnea, cardiovascular disease, C-reactive protein, total bilirubin, blood glucose, and urea. To use the nomogram, an individual patient's value is located on each variable axis, and a line is drawn upward to determine the number of points received for each variable value. The sum of these numbers is located on the Total Points axis, and a line is drawn downward to the survival axes to determine the likelihood of survival of 5-, 15-, and 30-day survival. 

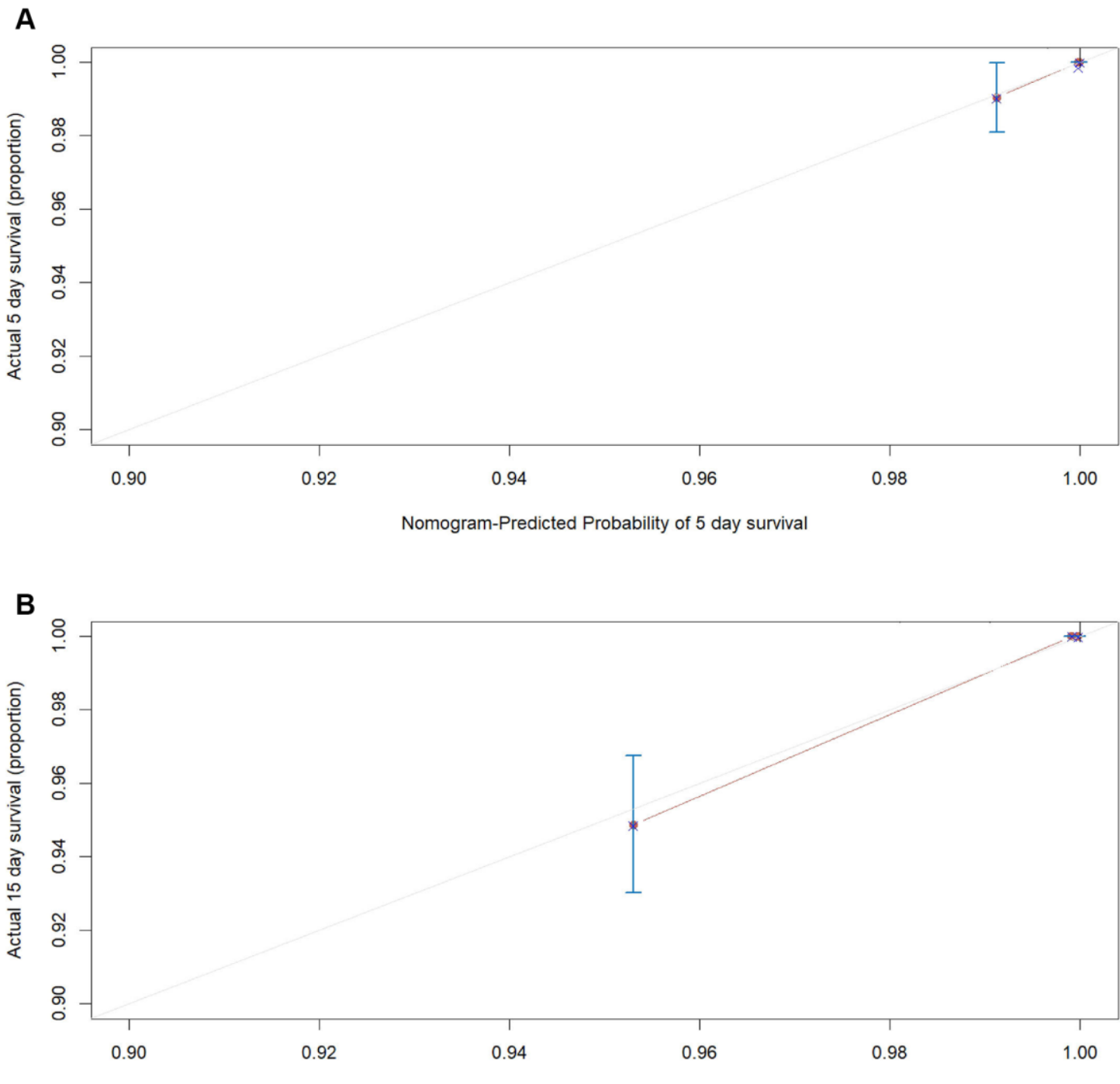

Nomogram-Predicted Probability of 15 day survival

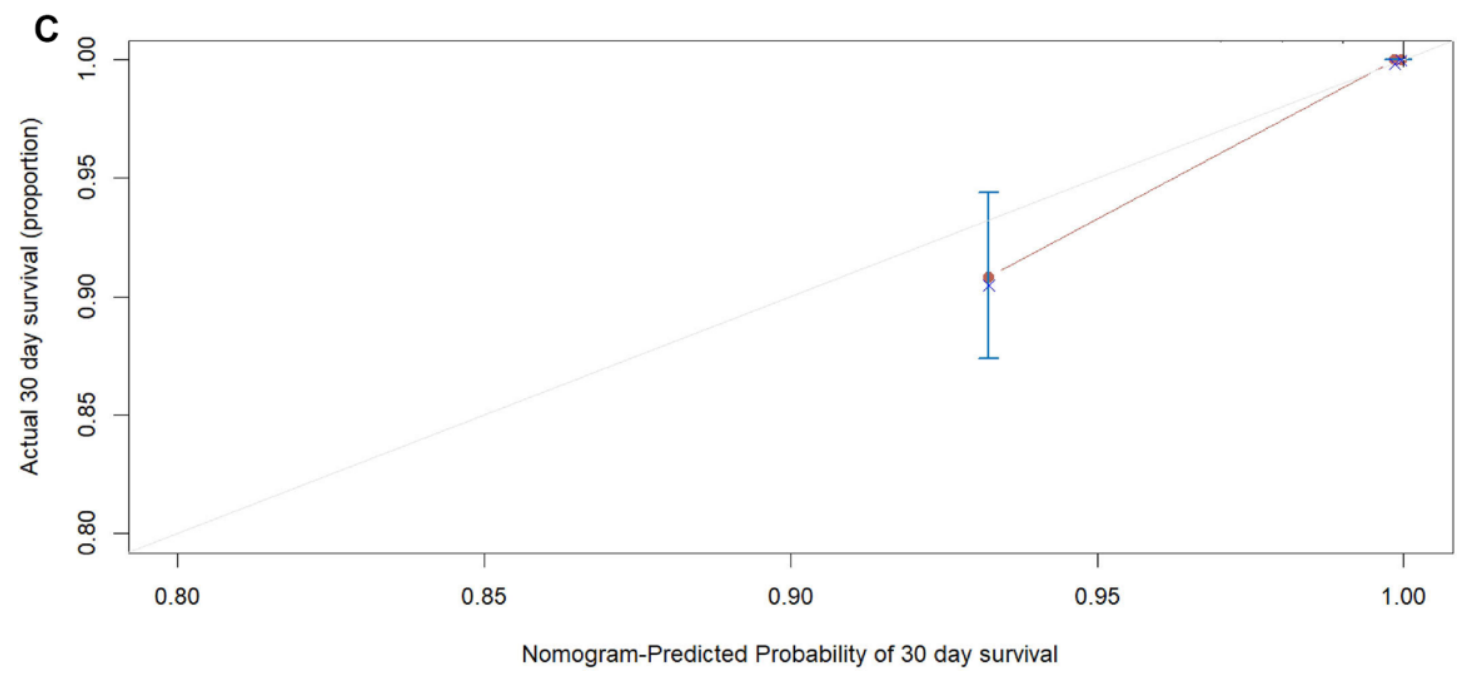

Figure 3. The calibration plots for the probability of in-hospital mortality of COVID-19 in the development cohort. Calibration plots of the nomogram predict (A) 5-day, (B) 15-day and (C) 30-day in-hospital mortality in COVID-19 patients in the development cohort. Nomogram-predicted probability of in-hospital mortality is plotted on the $x$-axis; actual in-hospital mortality is plotted on the $y$-axis. 

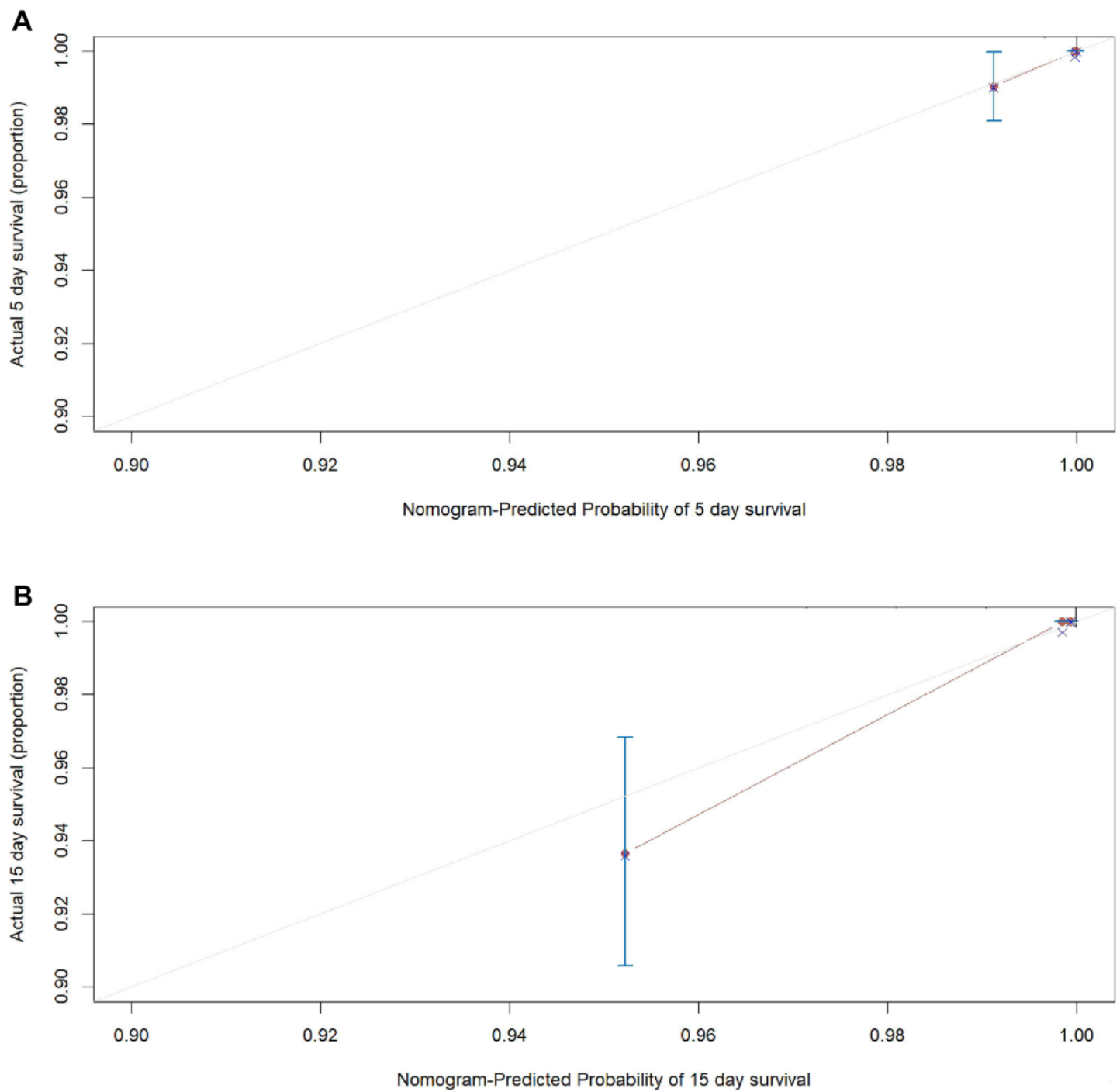

C

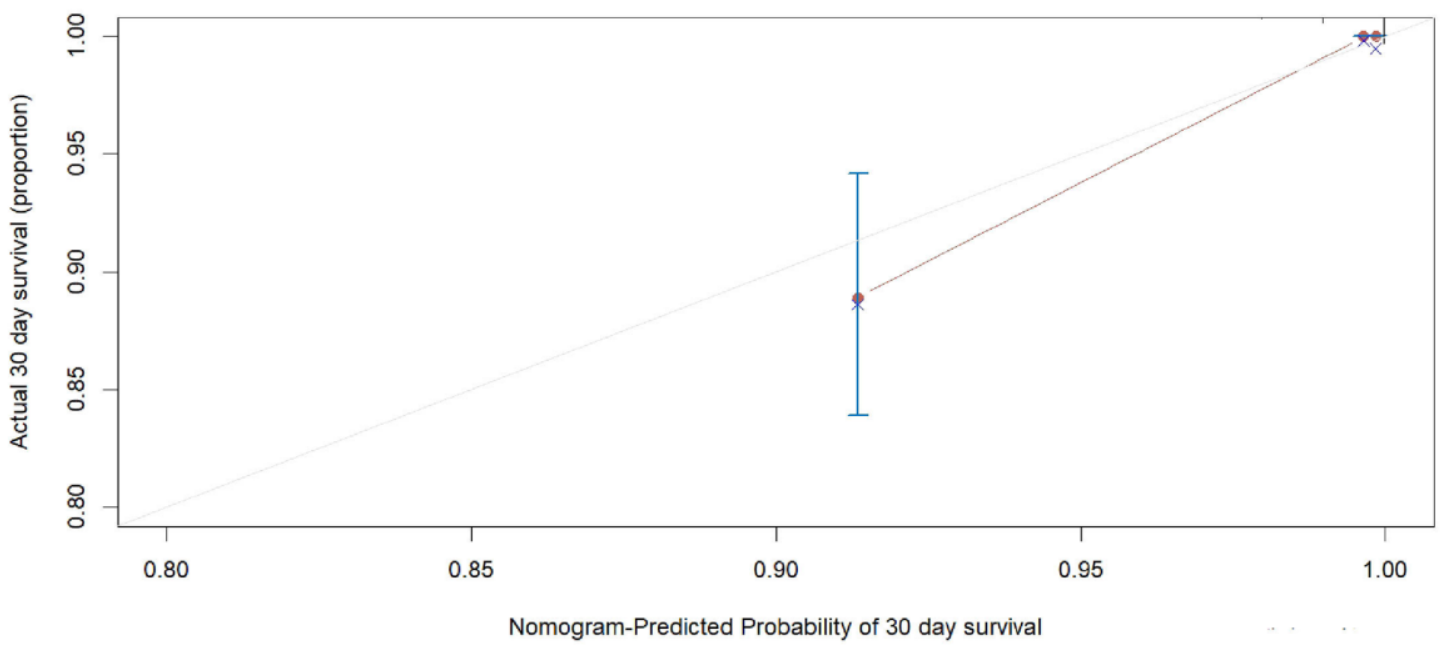

Figure 4. The calibration plots for the probability of in-hospital mortality of COVID-19 in internal validation cohort. Calibration plots of the nomogram predict (A) 5-day, (B) 15-day and (C) 30-day in-hospital mortality in COVID-19 patients in the validation cohort. Nomogram-predicted probability of in-hospital mortality is plotted on the $\mathrm{x}$-axis; actual in-hospital mortality is plotted on the $y$-axis. 

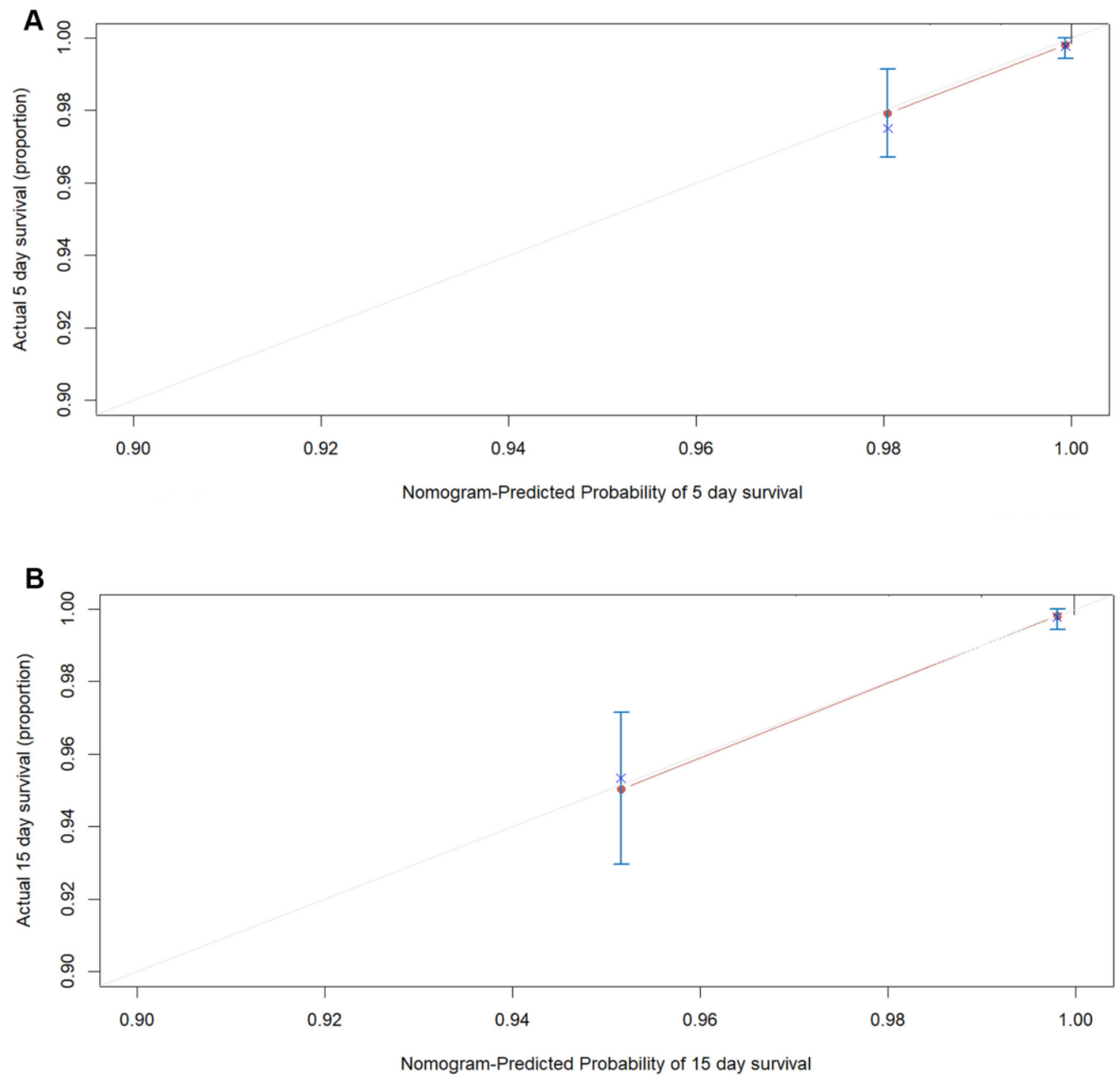

C

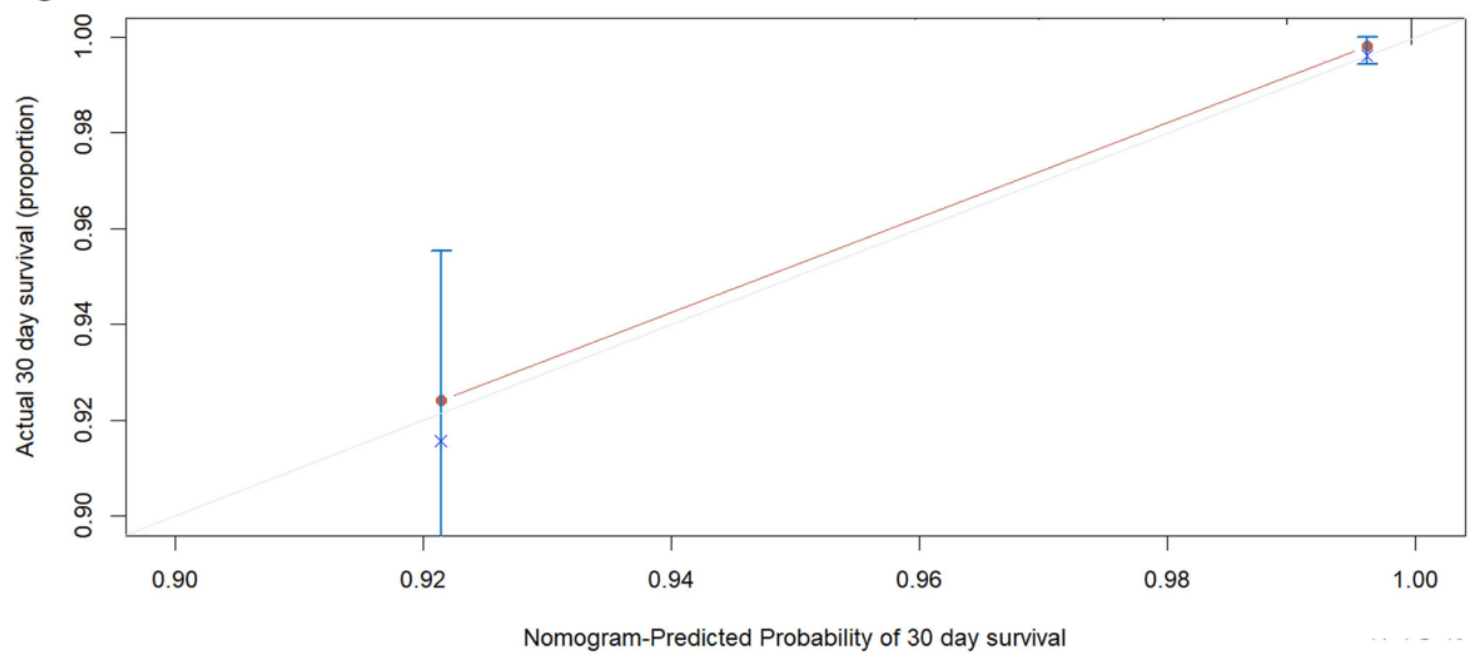

Figure 5. The calibration plots for the probability of in-hospital mortality of COVID-19 in external validation cohort. Calibration plots of the nomogram predict (A) 5-day, (B) 15-day and (C) 30-day in-hospital mortality in COVID-19 patients in the validation cohort. Nomogram-predicted probability of in-hospital mortality is plotted on the $\mathrm{x}$-axis; actual in-hospital mortality is plotted on the $y$-axis. 
Demographic, clinical, and laboratory parameters were included in prognostic models for COVID-19. The current model included eight predictors (age, severity at admission, dyspnea, cardiovascular disease, and levels of lactate dehydrogenase, total bilirubin, blood glucose, and urea). Of them, age and comorbidity with cardiovascular disease have been reported in previous models to be risk factors for either mortality or disease progression $[9,13-16]$. Severity at admission was mainly determined by $\mathrm{SpO} 2$ and $\mathrm{CT}$ imaging, as detailed before [17]. The prevalence of dyspnea is barely higher in patients who develop acute respiratory distress and have the poorest clinical outcomes, and was suggested to be a risk factor for predicting mortality in patients with COVID-19 $[18,19]$.

As for laboratory markers in the current model, the involvement of total bilirubin, blood glucose, urea, and lactate dehydrogenase indicates that involvement of multi-organ dysfunction represents a major predictor of in-hospital mortality for COVID-19 patients. In another recent nomogram, high direct bilirubin level was found to be an independent predictor of 28-day mortality in adult hospitalized patients with confirmed COVID-19 [7]. However, another study with a larger sample size found that AST abnormality, rather than bilirubin, was strongly associated with COVID-19 mortality risk [20]. A recent meta-analysis concluded that comorbid diabetes was associated with an increased risk of disease severity or death in Chinese COVID-19 patients, while it is still not clear to what extent diabetes independently contributes to the increased risk [21]. Besides, acute kidney injury is associated with severe infection and fatality in patients with COVID-19 [22]. The combination of blood urea nitrogen and D-Dimer were predictors of in-hospital mortality in 305 COVID-19 patients, with $27.9 \%$ mortality [23].

The current study provides a practical quantitative prognosis judgement tool (nomogram) for clinicians. We have the following strengths: First, sampling bias was avoided as much as possible by inclusion of all COVID-19 patients treated in the 2 designated hospitals, with the largest sample size to date. Second, the model showed good performance in both internal and external validations. Third, C-index and the calibration plot showed adequate calibration and discrimination capacity. Finally, we conducted the current study in strict compliance with the TRIPOD guideline, and PROBAST categorized it as at low risk of bias. Meanwhile, the current study has also several limitations. First, the retrospective study design limited the hierarchy of research evidence, and a prospective study is warranted to confirm the reliability of the findings. Second, missing data of some variables existed due to the emergency situations. However, the missing rate was of less than $10.0 \%$, and the missing values was imputed by EM method. Third, further validations from different hospitals or countries are warranted.

\section{CONCLUSIONS}

Conclusively, a novel prognostic nomogram for COVID-19 based on age, severity at admission, cardiovascular disease, and levels of lactate dehydrogenase, total bilirubin, blood glucose, and urea, was established and validated. It would be helpful for physicians to make optimal treatment decisions, conduct reasonable triage of patients, and avoid delays in treatment. Further studies are warranted to validate whether use of this prognostic nomogram will improve clinical care and patient outcomes of COVID-19.

\section{MATERIALS AND METHODS}

\section{Patients and study design}

The retrospective cohort study for prognosis model of COVID-19 was conducted according to the TRIPOD reporting guideline and the risk of bias was accessed using the PROBAST scales [24-27]. The study protocol was registered in the ChiCTR (http://www.chictr.org.cn, ChiCTR2000030256), and approved by the ethics committee of Wuhan Huoshenshan Hospital (HSSLL024) and Taikang-Tongji hospital (TKTJKY2020146) (Supplementary Materials). The informed consent was waived due to dealing with urgent public health concerns. A total of 3,022 COVID-19 cases from Wuhan Huoshenshan Hospital, and 1,064 COVID-19 cases from Taikang-Tongji hospital with relevant epidemiological and clinical data were included in this investigation. The diagnosis of COVID-19 patients was based on the World Health Organization interim guidance [28]. The endpoint of this study was in-hospital mortality of COVID-19 patients. The degree of severity of COVID-19 at admission was determined according to "Guidance for COVID-19 Prevention, Control, Diagnosis and Management" by National Health Commission of the People's Republic of China, which was divided into four categories: mild, ordinary, severe and critical (Supplementary Materials) [17]. Of the total 3,022 cases from Wuhan Huoshenshan Hospital, data of 1,780 cases recruited between February $3^{\text {rd }}$ and March $5^{\text {th }}$ was used for the development of the prognostic nomogram, while data for 1,242 cases recruited between March $5^{\text {th }}$ and April $10^{\text {th }}$ was used for the internal validation of the established prognostic nomogram. Further, data of 1,064 COVID-19 cases from Taikang-Tongji hospital was used for the external validation. 


\section{Data collection and entry}

Information of demographic characteristics and coexisting disorders was telephone-interviewed using a uniformed questionnaire by two trained physicians. The clinical symptoms, laboratory characteristics, and outcomes information were extracted from the electronic medical records. We double entered and validated the data using EpiData (version 3.1, EpiData Association, Odense, Denmark) software, and disputes were arbitrated by the expert committees composed of experts of respiratory and critical care medicine, and epidemiology.

\section{Construction of the prognostic nomogram}

All statistical analyses were conducted using the SAS statistical software (version 9.4; SAS Institute Inc., Cary, NC, USA) and the R software version 4.0.0 (Institute for Statistics and Mathematics, Vienna, Austria). $P$-value of $<0.05$ was considered statistically significant. Categorical variables were described using frequency rates and percentages, while continuous variables were described using the median/interquartile range (IQR) values. The missing values of all potential predictors (missing rate of less than 10.0\%) were imputed by expectation-maximization (EM) method. Univariate and multivariate Cox regression analysis was adopted for the estimation of hazard ratio (HR) and corresponding confidence interval (CI) of each variable. First, univariate Cox regression analysis was used to screen the potential prognostic factors which reached a $\mathrm{P}$ value of less than 0.05. Then, the independent prognostic factors were derived from a backward stepdown selection process in multivariate Cox regression model. Finally, a prognostic nomogram was formulated based on the results of multivariate analysis by using the rms package, according to the Akaike information criterion (AIC) [29].

\section{Validation and calibration of the prognostic nomogram}

The prognostic nomogram was subjected to 1,000 bootstrap resamples of the primary development cohort, an internal validation cohort, as well as an external validation cohort. The performance of the nomogram was measured by Harrel concordance index (C-index) and the calibration plot. The value of the $\mathrm{C}$-index, which assesses the discrimination of the model, ranges from 0.5 to 1.0 , and a larger $\mathrm{C}$-index means a more accurate prognostic model ( 0.5 indicating a random chance and 1.0 indicating a perfect ability to correctly discriminate the outcome with the model). During the validation of the prognostic nomogram, the total points of each patient in the validation cohort were calculated according to the established nomogram, then Cox regression in this cohort was performed using the total points as a factor, and finally, the C-index and calibration plot were derived based on the regression analysis.

\section{AUTHOR CONTRIBUTIONS}

MX and CG conceived and designed the study. MX, LL, FX, and CL drafted the paper and did the statistical analysis. LL, CL, FX, WP, LS, YH, ZY, JN, ZT, HC, ZJ, LS, PY, LY, NL, LY, SQ, JH, LM collected the data. All authors approved the final draft of the manuscript for publication.

\section{CONFLICTS OF INTEREST}

All authors declare no conflicts of interest.

\section{FUNDING}

The present study was funded by Outstanding Youth Science Foundation of Chongqing (cstc2020jcyjjqX0014), and the Science Foundation for Outstanding Young People of the Army Medical University (grant to Pro Xiangyu $\mathrm{Ma}$ and $\mathrm{Li} \mathrm{Li}$ ). The funders had no role in study design, data collection and analysis, decision to publish, or preparation of the manuscript.

\section{REFERENCES}

1. WHO. WHO Virtual press conference on COVID-19. 2020.

2. Xie J, Tong Z, Guan X, Du B, Qiu H, Slutsky AS. Critical care crisis and some recommendations during the COVID-19 epidemic in China. Intensive Care Med. 2020; 46:837-40. https://doi.org/10.1007/s00134-020-05979-7 PMID:32123994

3. Fang $X$, Li S, Yu H, Wang P, Zhang Y, Chen Z, Li Y, Cheng L, Li W, Jia H, Ma X. Epidemiological, comorbidity factors with severity and prognosis of COVID-19: a systematic review and meta-analysis. Aging (Albany NY). 2020; 12:12493-503. https://doi.org/10.18632/aging.103579 PMID: $\underline{32658868}$

4. Chen T, Wu D, Chen H, Yan W, Yang D, Chen G, Ma K, Xu D, Yu H, Wang H, Wang T, Guo W, Chen J, et al. Clinical characteristics of 113 deceased patients with coronavirus disease 2019: retrospective study. BMJ. 2020; 368:m1091. https://doi.org/10.1136/bmj.m1091 PMID: 32217556

5. Wu Z, McGoogan JM. Characteristics of and important lessons from the coronavirus disease 2019 (COVID-19) outbreak in China: summary of a report of 72314 
cases from the Chinese center for disease control and prevention. JAMA. 2020; 323:1239-42.

https://doi.org/10.1001/jama.2020.2648

PMID:32091533

6. Zhou F, Yu T, Du R, Fan G, Liu Y, Liu Z, Xiang J, Wang Y, Song B, Gu X, Guan L, Wei Y, Li H, et al. Clinical course and risk factors for mortality of adult inpatients with COVID-19 in Wuhan, China: a retrospective cohort study. Lancet. 2020; 395:1054-62.

https://doi.org/10.1016/S0140-6736(20)30566-3 PMID:32171076

7. Zhang S, Guo M, Duan L, Wu F, Hu G, Wang Z, Huang Q, Liao T, Xu J, Ma Y, Lv Z, Xiao W, Zhao Z, et al. Development and validation of a risk factor-based system to predict short-term survival in adult hospitalized patients with COVID-19: a multicenter, retrospective, cohort study. Crit Care. 2020; 24:438. https://doi.org/10.1186/s13054-020-03123-x PMID:32678040

8. Yan $L$, Zhang HT, Xiao $Y$, Wang $M$, Guo $Y$, Sun $C$, Tang $X$, Jing L, Li S, Zhang $M$, Xiao $Y$, Cao $H$, Chen $Y$, et al. Prediction of criticality in patients with severe Covid-19 infection using three clinical features: a machine learning-based prognostic model with clinical data in Wuhan. medRxiv. 2020. [Epub ahead of print]. https://doi.org/10.1101/2020.02.27.20028027

9. Bello-Chavolla OY, Bahena-López JP, Antonio-Villa NE, Vargas-Vázquez A, González-Díaz A, Márquez-Salinas A, Fermín-Martínez CA, Naveja JJ, Aguilar-Salinas CA. Predicting Mortality Due to SARS-CoV-2: A Mechanistic Score Relating Obesity and Diabetes to COVID-19 Outcomes in Mexico. J Clin Endocrinol Metab. 2020; 105:dgaa346.

https://doi.org/10.1210/clinem/dgaa346 PMID:32474598

10. Das AK, Mishra S, Saraswathy Gopalan S. Predicting CoVID-19 community mortality risk using machine learning and development of an online prognostic tool. PeerJ. 2020; 8:e10083. https://doi.org/10.7717/peeri.10083 PMID:33062451

11. Yuan M, Yin W, Tao Z, Tan W, Hu Y. Association of radiologic findings with mortality of patients infected with 2019 novel coronavirus in Wuhan, China. PLoS One. 2020; $15: \mathrm{e} 0230548$.

https://doi.org/10.1371/journal.pone.0230548 PMID:32191764

12. Wynants L, Van Calster B, Collins GS, Riley RD, Heinze G, Schuit E, Bonten MM, Damen JA, Debray TP, De Vos $M$, Dhiman $\mathrm{P}$, Haller MC, Harhay MO, et al. Prediction models for diagnosis and prognosis of covid-19 infection: systematic review and critical appraisal. BMJ. 2020; 369:m1328. https://doi.org/10.1136/bmj.m1328 PMID:32265220

13. Colombi D, Bodini FC, Petrini M, Maffi G, Morelli N, Milanese G, Silva M, Sverzellati N, Michieletti E. Wellaerated lung on admitting chest $\mathrm{CT}$ to predict adverse outcome in COVID-19 pneumonia. Radiology. 2020; 296:E86-96.

https://doi.org/10.1148/radiol.2020201433 PMID:32301647

14. Ji D, Zhang D, Xu J, Chen Z, Yang T, Zhao P, Chen G, Cheng G, Wang Y, Bi J, Tan L, Lau G, Qin E. Prediction for progression risk in patients with COVID-19 pneumonia: the CALL score. Clin Infect Dis. 2020; 71:1393-99.

https://doi.org/10.1093/cid/ciaa414 PMID:32271369

15. Barda N, Riesel D, Akriv A, Levi J, Finkel U, Yona G, Greenfeld D, Sheiba S, Somer J, Bachmat E, Rothblum GN, Shalit $U$, Netzer $D$, et al. Performing risk stratification for COVID-19 when individual level data is not available - the experience of a large healthcare organization. medRxiv. 2020. [Epub ahead of print]. https://doi.org/10.1101/2020.04.23.20076976

16. Chen $R$, Liang $W$, Jiang $M$, Guan $W$, Zhan $C$, Wang $T$, Tang C, Sang L, Liu J, Ni Z, Hu Y, Liu L, Shan H, et al, and Medical Treatment Expert Group for COVID-19. Risk factors of fatal outcome in hospitalized subjects with coronavirus disease 2019 from a nationwide analysis in China. Chest. 2020; 158:97-105.

https://doi.org/10.1016/j.chest.2020.04.010 PMID:32304772

17. Commission HC. The New Coronavirus Pneumonia Prevention and Control Guidelines of China. 2020.

18. Shi L, Wang $Y$, Wang $Y$, Duan $G$, Yang $H$. Dyspnea rather than fever is a risk factor for predicting mortality in patients with COVID-19. J Infect. 2020; 81:647-79. https://doi.org/10.1016/i.jinf.2020.05.013 PMID:32417316

19. Allali G, Marti C, Grosgurin O, Morélot-Panzini C, Similowski T, Adler D. Dyspnea: the vanished warning symptom of COVID-19 pneumonia. J Med Virol. 2020; 92:2272-73. https://doi.org/10.1002/imv.26172 PMID:32530534

20. Lei $F$, Liu YM, Zhou F, Qin JJ, Zhang P, Zhu L, Zhang XJ, Cai J, Lin L, Ouyang S, Wang $X$, Yang C, Cheng $X$, et al. Longitudinal association between markers of liver injury and mortality in COVID-19 in China. Hepatology. 2020; 72:389-98.

https://doi.org/10.1002/hep.31301 PMID:32359177

21. Guo L, Shi Z, Zhang Y, Wang C, Do Vale Moreira NC, Zuo $\mathrm{H}$, Hussain A. Comorbid diabetes and the risk of disease severity or death among 8807 COVID-19 
patients in China: a meta-analysis. Diabetes Res Clin Pract. 2020; 166:108346.

https://doi.org/10.1016/j.diabres.2020.108346

PMID:32710998

22. Shao M, Li X, Liu F, Tian T, Luo J, Yang Y. Acute kidney injury is associated with severe infection and fatality in patients with COVID-19: a systematic review and metaanalysis of 40 studies and 24,527 patients. Pharmacol Res. 2020; 161:105107. https://doi.org/10.1016/j.phrs.2020.105107 PMID: $\underline{32739424}$

23. Cheng $A$, Hu L, Wang $Y$, Huang $L$, Zhao $L$, Zhang $C$, Liu $X$, Xu R, Liu F, Li J, Ye D, Wang T, Lv Y, Liu Q. Diagnostic performance of initial blood urea nitrogen combined with D-dimer levels for predicting in-hospital mortality in COVID-19 patients. Int J Antimicrob Agents. 2020; 56:106110.

https://doi.org/10.1016/j.ijantimicag.2020.106110 PMID:32712332

24. Collins GS, Reitsma JB, Altman DG, Moons KG. Transparent Reporting of a multivariable prediction model for Individual Prognosis or Diagnosis (TRIPOD): the TRIPOD statement. Ann Intern Med. 2015; 162:55-63.

https://doi.org/10.7326/M14-0697 PMID:25560714

25. Moons KG, Wolff RF, Riley RD, Whiting PF, Westwood M, Collins GS, Reitsma JB, Kleijnen J, Mallett S. PROBAST: a tool to assess risk of bias and applicability of prediction model studies: explanation and elaboration. Ann Intern Med. 2019; 170:W1-33. https://doi.org/10.7326/M18-1377 PMID:

26. Wolff RF, Moons KG, Riley RD, Whiting PF, Westwood M, Collins GS, Reitsma JB, Kleijnen J, Mallett S, and PROBAST Groupt. PROBAST: a tool to assess the risk of bias and applicability of prediction model studies. Ann Intern Med. 2019; 170:51-58. https://doi.org/10.7326/M18-1376 PMID: 30596875

27. Moons KG, Altman DG, Reitsma JB, Ioannidis JP, Macaskill P, Steyerberg EW, Vickers AJ, Ransohoff DF, Collins GS. Transparent reporting of a multivariable prediction model for individual prognosis or diagnosis (TRIPOD): explanation and elaboration. Ann Intern Med. 2015; 162:W1-73. https://doi.org/10.7326/M14-0698 PMID:25560730

28. World Health Organization. Clinical management of severe acute respiratory infection (SARI) when COVID19 disease is suspected: interim guidance. 2020. WHO. https://apps.who.int/iris/handle/10665/331446

29. Harrell FE Jr, Lee KL, Mark DB. Multivariable prognostic models: issues in developing models, evaluating assumptions and adequacy, and measuring and reducing errors. Stat Med. 1996; 15:361-87. https://doi.org/10.1002/(SICI)10970258(19960229)15:4<361::AID-SIM168>3.0.CO;2-4 PMID:8668867 


\section{SUPPLEMENTARY MATERIALS}

\section{The grading criteria for the severity of COVID-19:}

(1) mild type: patients with mild clinical symptoms and no pulmonary changes on CT imaging;

(2) common type: patients with symptoms of fever and signs of respiratory infection, and having pneumonia changes on CT imaging;

(3) severe type: patients presenting with any one of the following conditions: a. respiratory distress, respiratory rate $\geq 30 / \mathrm{min} ; b$. oxygen saturation of finger $\leq 93 \%$ in resting condition; c. arterial partial pressure of oxygen $(\mathrm{PaO} 2)$ /oxygen concentration $(\mathrm{FiO} 2) \leq 300 \mathrm{mmHg}$ $(1 \mathrm{mmHg}=0.133 \mathrm{kPa}) ; \mathrm{d}$. The clinical symptoms are progressively worsening, and lung imaging shows that the lesion has progressed significantly>50\% within 24 to 48 hours;

(4) critical type: patients meeting any one of the following criteria: a. respiratory failure requiring mechanical ventilation; b. shock; c. concomitant failure of other organs and requirement for intensive care unit (ICU) monitoring and treatment.

\section{The relevant ethical review materials}

Proposal Title: Epidemiological features, clinical characteristics and prognosis of patients with novel coronavirus pneumonia

\section{Principal investigator: Guoqiang Cao}

Study design: observational study

\section{Key requirements:}

1. Subjects have the right to be informed about the health effects of the research and the results that can be obtained, and sign informed consent.

2. Each participant's demographic form and consent form will be stored separately in a secure location.

3. Transcription will be carried out in a private space. All personal identification information will be removed or changed during transcription.

4. Digital copies of the files will be encrypted, password protected and stored securely.

5. Research should comply with the Declaration of Helsinki. 